\title{
Molecular gas around low-luminosity AGN in late-type spirals
}

\author{
T. Böker ${ }^{1}$, E. Schinnerer ${ }^{2}$, and U. Lisenfeld ${ }^{3}$
}

\author{
1 Research and Scientific Support Department, European Space Agency, Keplerlaan 1, 2200 AG Noordwijk, The Netherlands \\ e-mail: tboeker@rssd.esa.int \\ 2 Max-Planck-Institute for Astronomy, Königstuhl 17, 69117 Heidelberg, Germany \\ 3 Departamento de Física Teórica y del Cosmos, Facultad de Ciencias, Universidad de Granada, Spain
}

Received 15 May 2011 / Accepted 26 July 2011

\begin{abstract}
We have studied the molecular gas in the vicinity of low-luminosity active galactic nuclei (AGNs) in three bulge-less spiral galaxies: NGC 1042, NGC 4178, and NGC 4395. The (1-0) and (2-1) transitions of gaseous carbon monoxide (CO) are clearly detected within the central kpc of all three galaxies. In the case of NGC 4395, this constitutes the first reported detection of CO. In general, the CO emission is faint, as may be expected from their less-than-spectacular star formation activity. Interestingly, however, both face-on galaxies in our sample (which allow an unimpeded view of their nucleus) show an elevated intensity ratio $\mathrm{CO}(2-1) / \mathrm{CO}(1-0)$ when compared to similar late-type spirals without an AGN. We discuss that this is unlikely due to a very compact CO source. Instead, we speculate that even energetically weak AGN can impact the physical state of the surrounding gas. We do not detect any tracers of dense molecular gas such as $\mathrm{HCN}$ or $\mathrm{HCO}+$, but the sensitivity of our observations allows us to establish upper limits that lie at the low end of the range observed in more energetic AGN. The derived gas density is less than $n_{\mathrm{H}_{2}} \approx 2 \times 10^{3} \mathrm{~cm}^{-3}$ which is significantly lower than in most other nearby galaxies. The scarcity of dense gas suggests that the conditions for star formation are poor in these nuclei.
\end{abstract}

Key words. ISM: molecules - galaxies: spiral - galaxies: ISM - galaxies: nuclei

\section{Introduction}

Recent studies have demonstrated that supermassive black holes (SMBHs) reside in the nuclei of most bulge-dominated galaxies, and that the mass of the $\mathrm{SMBH}, M_{\mathrm{BH}}$, is strongly correlated with various properties of the host spheroid (e.g. Gebhardt et al. 2000; Ferrarese \& Merritt 2000). This discovery has launched numerous speculations that the formation and evolution of galaxies and their SMBHs are fundamentally linked, and that perhaps the presence of a bulge is a necessary ingredient for a black hole to form and grow. Indeed, M 33, the most nearby example of a truly bulgeless disk galaxy shows no evidence of a SMBH, and the upper limit on the black hole mass (determined from stellar dynamics) is significantly below that predicted by the $M_{\mathrm{BH}}-\sigma$ relation for early-type galaxies (Gebhardt et al. 2001). On the other hand, the galaxy NGC 4395 shows no evidence for a bulge either, yet it has long been known to harbor an active nucleus (Filippenko \& Sargent 1989). Until recently, however, this galaxy was the only known example of an accreting black hole in a bulgeless disk, leaving open the possibility that it is just an anomaly.

Over the past few years, however, a number of similar cases have been found that have put into question whether black holes truly require a bulge to form or grow. These discoveries came from optical spectroscopy of nuclear star clusters (NSCs) that revealed emission line ratios and profiles indicative of nuclear activity (NGC 1042; Shields et al. 2008), the detection of high-excitation mid-infrared lines such as [NeV] (NGC 4178; Satyapal et al. 2009), or a combination of both (NGC 3621; Satyapal et al. 2007; Barth et al. 2009). In all cases, the AGN is energetically weak, and the inferred SMBH masses are at the lower end of the mass range probed by the established $M_{\mathrm{BH}}-\sigma$ relation, typically below $10^{6} M_{\odot}$. For these reasons, they are often referred to as "mini-AGN".

While the number of such "mini-AGN" is likely to increase over the coming years, it seems that they are indeed rare, i.e. the AGN fraction is lower in "pure" disks than in more bulgedominated galaxies (Satyapal et al. 2009). A clue for why this may be so comes from the recent finding that the nuclei of bulgeless disks are often occupied by a compact, massive star cluster (e.g. Böker et al. 2002). It has been speculated that the formation of such a NSC can inhibit the growth of a SMBH because gas is expelled from the nucleus by the mechanical feedback from massive stars (Schinnerer et al. 2008; Nayakshin et al. 2009). While the relative importance of the SMBH indeed appears to drop strongly towards lower spheroid masses, it is interesting to note that all "mini-AGN" known so far are located in galaxies with an NSC (Satyapal et al. 2009).

The molecular gas in the vicinity of a galaxy nucleus is the raw material to support both star formation and (directly or indirectly) SMBH growth. Conversely, either type of nuclear activity influences the chemical composition and excitation conditions of the surrounding molecular gas (e.g. Sternberg et al. 1994; Kohno 2005; Graciá-Carpio et al. 2006; Krips et al. 2008). Studies of the molecular gas, therefore, can reveal important information about the relative importance of both phenomena. For example, the flux ratio between ${ }^{12} \mathrm{CO}(1-0)$ and the dense gas tracers $\mathrm{HCN}(1-0)$, and $\mathrm{HCO}^{+}(1-0)$ is a useful indicator for the strength of an AGN (Kohno 2005), with considerably higher HCN/CO and $\mathrm{HCO}^{+} / \mathrm{CO}$ flux ratios in AGN than in starbursts. This questions the validity of the $\mathrm{HCN}$ intensity as a tracer for SF activity (Gao \& Solomon 2004b,a; Graciá-Carpio et al. 2006) in AGN sources. Models of X-ray dominated regions have been evoked to explain the enhanced $\mathrm{HCN}$ emission in AGN suggesting that 
Table 1. Target galaxies.

\begin{tabular}{lccccccc}
\hline \hline $\begin{array}{l}(1) \\
\text { Galaxy }\end{array}$ & $\begin{array}{c}(2) \\
\text { RA }\end{array}$ & $\begin{array}{c}(3) \\
\mathrm{Dec}\end{array}$ & $\begin{array}{c}(4) \\
\mathrm{Mpc}\end{array}$ & $\begin{array}{c}(5) \\
v_{\text {sys }} \\
{\left[\mathrm{km} \mathrm{s}^{-1}\right]}\end{array}$ & $\begin{array}{c}(6) \\
W_{20} \\
{\left[\mathrm{~km} \mathrm{~s}^{-1}\right]}\end{array}$ & $\begin{array}{c}L_{\mathrm{BgN}}^{\mathrm{AGN}} \\
{[\mathrm{erg} / \mathrm{s}]}\end{array}$ & $\begin{array}{c}(8) \\
M_{\mathrm{BH}} \\
{\left[M_{\odot}\right]}\end{array}$ \\
\hline NGC 1042 & $02^{\mathrm{h}} 40^{\mathrm{m}} 23.8^{\mathrm{s}}$ & $-08: 26: 00.0$ & 16.7 & 1377 & 124 & $8 \times 10^{39}$ & $60 \lesssim M \lesssim 3 \times 10^{6}$ \\
NGC 4178 & $12^{\mathrm{h}} 12^{\mathrm{m}} 46.3^{\mathrm{s}}$ & $10: 51: 54.0$ & 16.8 & 381 & 291 & $8 \times 10^{41}$ & $M \gtrsim 6000$ \\
NGC 4395 & $12^{\mathrm{h}} 25^{\mathrm{m}} 48.8^{\mathrm{s}}$ & $33: 32: 48.0$ & 4.1 & 318 & 140 & $5.4 \times 10^{40}$ & $(3.6 \pm 1.1) \times 10^{5}$ \\
\hline
\end{tabular}

Notes. Systemic velocity (Col. 5) and HI line width at 20\% level (Col. 6) are from Fisher \& Tully (1981). The distances to NGC 1042 and NGC 4178 are from Tully (1988), that to NGC 4395 from Thim et al. (2004). References for AGN properties and SMBH masses in Cols. (7) and (8) are as follows: NGC 1042: Shields et al. (2008), NGC 4178: Satyapal et al. (2009), NGC 4395: Peterson et al. (2005).
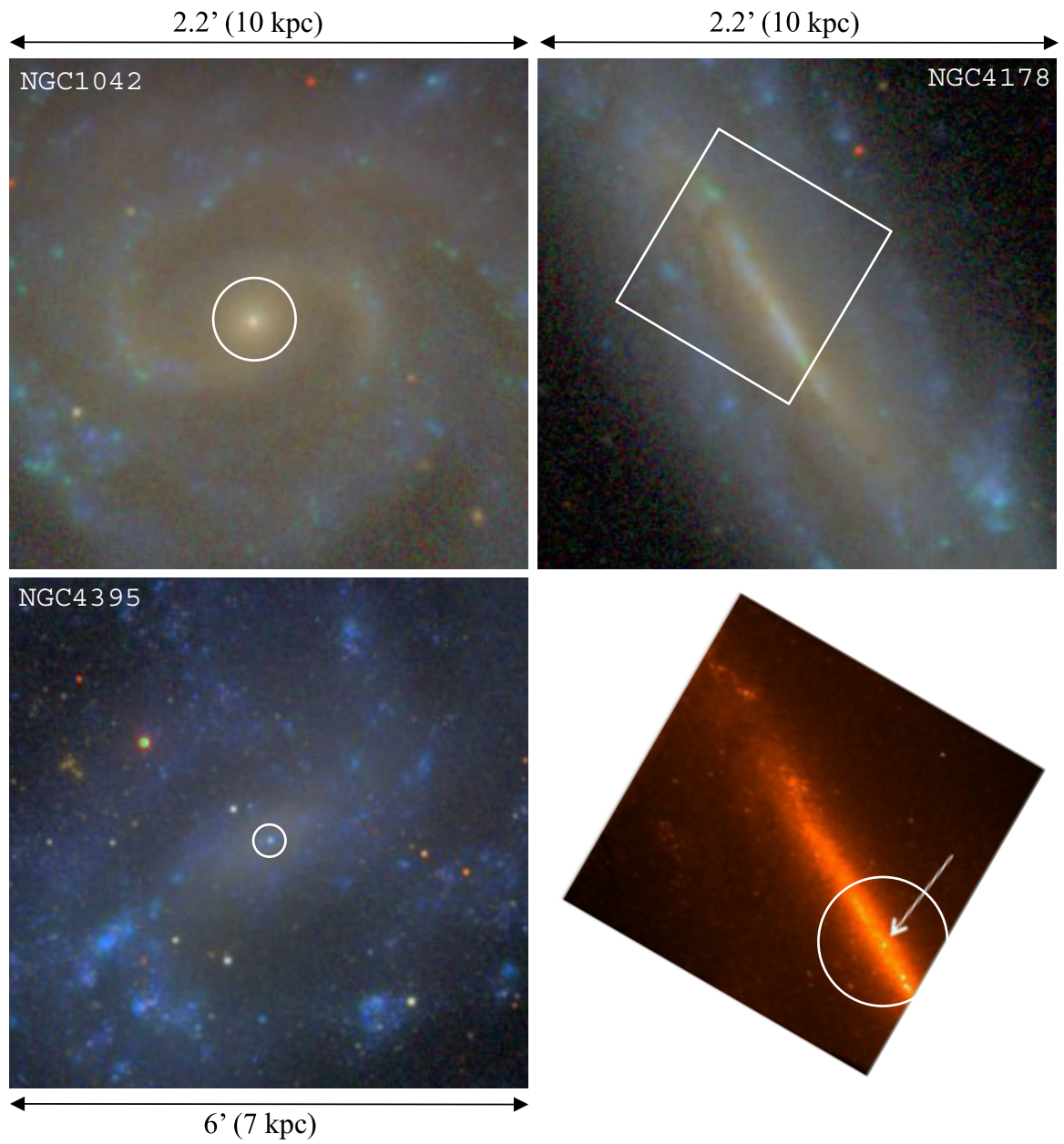

Fig. 1. Optical/near-infrared images of our target galaxies. Approximate image sizes are indicated, their orientation, however, is arbitrary. All three galaxies clearly harbor a nuclear star cluster (indicated by an arrow in the case of NGC 4178). The size and location of the IRAM beam used for the observations described here $\left(21^{\prime \prime}\right.$ at $\left.115 \mathrm{GHz}\right)$ is indicated by the circles.

the AGN itself is altering the chemical properties of the surrounding ISM (e.g. Meijerink \& Spaans 2005; Meijerink et al. 2006, 2007).

In this paper, we investigate the molecular gas content in all three "mini-AGN" observable from the northern hemisphere, i.e. NGC 1042, NGC 4178, and NGC 4395, with the goal of comparing them to more luminous AGN on the one hand, and inactive late-type disks on the other. Some properties of the target galaxies and their AGN are summarized in Table 1. In addition to an $\mathrm{SMBH}$, all three galaxies also host a prominent NSC, as can be seen in Fig. 1.

Following this introduction, we briefly describe the observations and data reduction procedure in Sect. 2, present and discuss our findings in Sect. 3, and summarize their implications in Sect. 4.

\section{Observations and data reduction}

All data discussed here were obtained during the period May 23-25, 2010 with the $30 \mathrm{~m}$ millimeter-wave telescope on Pico Veleta (Spain) operated by the Institut de Radio Astronomie Millimétrique (IRAM). We used the EMIR receivers, with the autocorrelator WILMA as a backend. This setup yields a resolution of $2 \mathrm{MHz}$ and a bandwidth of $3.7 \mathrm{GHz}$. All observations were performed in wobbler-switching mode, with a throw in azimuth of $240^{\prime \prime}$. The telescope pointing was checked on a nearby quasar about every $90 \mathrm{~min}$. 
T. Böker et al.: Molecular gas around low-luminosity AGN in late-type spirals

Table 2. Summary of measured velocity-integrated line intensities and derived molecular gas masses.

\begin{tabular}{lcccccc}
\hline \hline (1) & $(2)$ & $(3)$ & $(4)$ & $(5)$ & $(6)$ & $(7)$ \\
& $\begin{array}{c}I_{10} \\
{\left[\mathrm{~K} \mathrm{~km} \mathrm{~s}^{-1}\right]}\end{array}$ & $\begin{array}{c}I_{21} \\
{\left[\mathrm{~K} \mathrm{~km} \mathrm{~s}^{-1}\right]}\end{array}$ & $\begin{array}{c}I_{\mathrm{HCN}} \\
{\left[\mathrm{K} \mathrm{km} \mathrm{s}^{-1}\right]}\end{array}$ & $\begin{array}{c}I_{\mathrm{HCO}+} \\
{\left[\mathrm{K} \mathrm{km} \mathrm{s}^{-1}\right]}\end{array}$ & $\begin{array}{c}I_{13} \mathrm{CO} \\
{\left[\mathrm{K} \mathrm{km} \mathrm{s}^{-1}\right]}\end{array}$ & $\begin{array}{c}M_{\mathrm{H}_{2}} \\
{\left[10^{6} M_{\odot}\right]}\end{array}$ \\
\hline NGC 1042 & $2.26 \pm 0.11$ & $4.10 \pm 0.21$ & $<0.13$ & $<0.11$ & n.o. & 27.0 \\
NGC 4178 & $3.78 \pm 0.13$ & $3.19 \pm 0.18$ & $<0.10$ & $<0.07$ & $<0.30$ & 45.6 \\
NGC4395 $(0,0)$ & $0.23 \pm 0.04$ & $0.50 \pm 0.05$ & n.o. & n.o. & n.o. & 0.2 \\
NGC4395 $(15,0)$ & $<0.26$ & $<0.29$ & n.o. & n.o. & n.o. & $<0.2$ \\
NGC4395 (-15,0) & $<0.25$ & $<0.25$ & n.o. & n.o. & n.o. & $<0.2$ \\
NGC4395 $(0,15)$ & $<0.31$ & $<0.40$ & n.o. & n.o. & n.o. & $<0.2$ \\
NGC4395 (0,-15) & $<0.24$ & $<0.31$ & n.o. & n.o. & n.o. & $<0.2$ \\
\hline
\end{tabular}

Notes. The entry n.o. stands for "not observed". Upper limits for the line intensities were derived as described in Sect. 3.1.

All observations were carried out in good weather conditions, the mean system temperatures being $320 \mathrm{~K}$ on the $T_{\mathrm{A}}^{*}$ scale for the $\mathrm{CO}(1-0)$ and $\mathrm{CO}(2-1)$ transitions, and $105 \mathrm{~K}$ for the lowfrequency observations at $90 \mathrm{GHz}$. The main beam efficiencies were $0.81(90 \mathrm{GHz}), 0.77(115 \mathrm{GHz})$, and $0.58(230 \mathrm{GHz})$, with half-power beam widths of about $28^{\prime \prime}(90 \mathrm{GHz}), 21^{\prime \prime}(115 \mathrm{GHz})$, and $11^{\prime \prime}(230 \mathrm{GHz})$.

Data reduction was straightforward: the spectra for each position were averaged. Only zero-order baselines (i.e. continuum levels) were subtracted to obtain the final spectra. We observed the central position of all three galaxies in the ${ }^{12} \mathrm{CO}(1-0)$ and ${ }^{12} \mathrm{CO}(2-1)$ transitions at $115 \mathrm{GHz}$ and $230 \mathrm{GHz}$ simultaneously, and in both polarizations. In order to cover comparable physical areas in all three galaxies, we also carried out a 4-point mapping of NGC 4395 in both CO lines, using offsets of $\pm 15^{\prime \prime}$ from the central position in both RA and Dec.

In addition, we attempted to observe the lines of $\mathrm{HCN}(1-0)$ (rest frequency $88.631 \mathrm{GHz}), \mathrm{HCO}+(1-0)(89.189 \mathrm{GHz})$, and HNC(1-0) (90.979 GHz) in NGC 1042 and NGC 4178. For this purpose, we centered the receiver bandwidth at $89.1665 \mathrm{GHz}$ and $89.5665 \mathrm{GHz}$, respectively, to compensate for the recession velocity of the galaxies. Finally, we also targeted the isotopic line ${ }^{13} \mathrm{CO}(2-1)$ of $\mathrm{NGC} 4178$ at $1 \mathrm{~mm}$.

\section{Results and discussion}

\subsection{Integrated line intensities and upper limits}

The ${ }^{12} \mathrm{CO}(1-0)$ and ${ }^{12} \mathrm{CO}(2-1)$ spectra for the central position of all three galaxies are presented in Fig. 2. Both lines are clearly detected in all three galaxies. We derive the velocity-integrated line intensities via two different methods: first, we simply sum up all channels with significant emission. We use the same velocity window for ${ }^{12} \mathrm{CO}(1-0)$ and ${ }^{12} \mathrm{CO}(2-1)$ because the underlying assumption is that both lines originate from the same gas distribution. The integration windows are indicated by solid horizontal lines in Fig. 2.

We also fit Gaussian profiles to the lines, which yields an alternative estimate for the line intensities. These fits are also shown in Fig. 2. The agreement between the two methods is very good: the derived line intensities agree to within $5 \%$. In Table 2 , we list the average of the two methods.

A comparison with other $\mathrm{CO}$ observations in the literature yields the following: NGC 1042 was observed by Braine et al. (1993) who measure $I_{10}=2.9 \pm 0.3 \mathrm{~K} \mathrm{~km} \mathrm{~s}^{-1}$ with the IRAM $30 \mathrm{~m}$ telescope, in reasonable agreement with our results. NGC 4178 was observed by Kenney \& Young (1988) who report a (marginal) detection of $I_{10}=0.9 \pm 0.3 \mathrm{~K} \mathrm{~km} \mathrm{~s}^{-1}$. For the same galaxy, Boselli et al. (1995) report $I_{10}=1.6 \pm 0.5 \mathrm{~K} \mathrm{~km} \mathrm{~s}^{-1}$, observed with the SEST $15 \mathrm{~m}$. The fact that $I_{10}$ is significantly smaller over the $43^{\prime \prime}$ SEST beam than what we measure with the IRAM telescope may indicate that the $\mathrm{CO}$ emission is less extended than the SEST beam. On the other hand, it is surprising that Komugi et al. (2008) only give an upper limit of $I_{10}<3.4 \mathrm{~K} \mathrm{~km} \mathrm{~s}^{-1}$ over the $16^{\prime \prime}$ beam of the Nobeyama $45 \mathrm{~m}$ telescope. Lastly, NGC 4395 has, to our knowledge, not been detected in $\mathrm{CO}$ before.

In Fig. 2, we do not show the $\mathrm{CO}$ spectra for the off-nuclear pointings in NGC 4395, because none of them shows any significant $\mathrm{CO}$ emission. We list in Table 2 conservative upper limits for the ${ }^{12} \mathrm{CO}(1-0)$ and ${ }^{12} \mathrm{CO}(2-1)$ lines, derived as $I_{\mathrm{CO}}<$ $3 \mathrm{rms} \sqrt{\Delta_{\mathrm{V}} \cdot \delta_{\mathrm{v}}}$ where $\mathrm{rms}$ is the rms noise (per channel) of the spectrum in question, $\Delta_{\mathrm{V}}$ is the integration window of the $\mathrm{CO}$ lines detected in the nucleus, and $\delta_{\mathrm{v}}$ is the velocity resolution, i.e. the channel width.

We also did not detect significant emission from $\mathrm{HCN}$, $\mathrm{HCO}+(1-0)$, or ${ }^{13} \mathrm{CO}(2-1)$ in any of the (observed) nuclei, the corresponding $3 \sigma$ upper limits are reported in Cols. 4-6 of Table 2. We also searched the entire receiver bandwidth of 3.7 GHz for possible detections of other emission lines; no lines above the limits reported for the above lines were detected.

\subsection{Estimates of molecular gas masses}

We can estimate the mass of molecular hydrogen contained within the area covered by the IRAM beam by using the generic Galactic conversion factor of $X=2.3 \times 10^{20} \mathrm{~cm}^{-2}\left(\mathrm{~K} \mathrm{~km} \mathrm{~s}^{-1}\right)^{-1}$ (Strong et al. 1988), which yields:

$M_{\mathrm{H}_{2}}=97 D^{2} I_{10} \Theta^{2} \quad\left[M_{\odot}\right]$

when assuming a Gaussian beam with solid angle $1.13 \cdot \Theta^{2}$ (for details, see Böker et al. 2003). Here, $D$ is the galaxy distance measured in Mpc, $\Theta$ is the half power beam width of the $30 \mathrm{~m}$ telescope $\left(21^{\prime \prime}\right)$, and $I_{10}$ the velocity integrated ${ }^{12} \mathrm{CO}(1-0)$ intensity in $\mathrm{K} \mathrm{km} \mathrm{s}^{-1}$. The resulting $\mathrm{H}_{2}$ masses for the three target galaxies are listed in Col. 7 of Table 2. The gas masses are rather low, especially in NGC 4395 where the entire $\mathrm{H}_{2}$ mass could be contained in a single giant molecular cloud (GMC) complex.

This conclusion is independent of the use of the generic conversion factor, because the galaxies studied here are neither extremely faint, nor have extreme metallicities. For example, the oxygen abundance in the disk of NGC 4395, the faintest of our targets, has been reported by Pilyugin (2001) to be $12+\log (\mathrm{O} / \mathrm{H})=8.3-8.4$, or about one third of the solar value (8.76, Caffau et al. 2008). Assuming that the nuclear region has at least this abundance, using the metallicity-dependent conversion factor proposed by Boselli et al. (2002) would increase the derived gas mass by no more than a factor of 2.5 . 

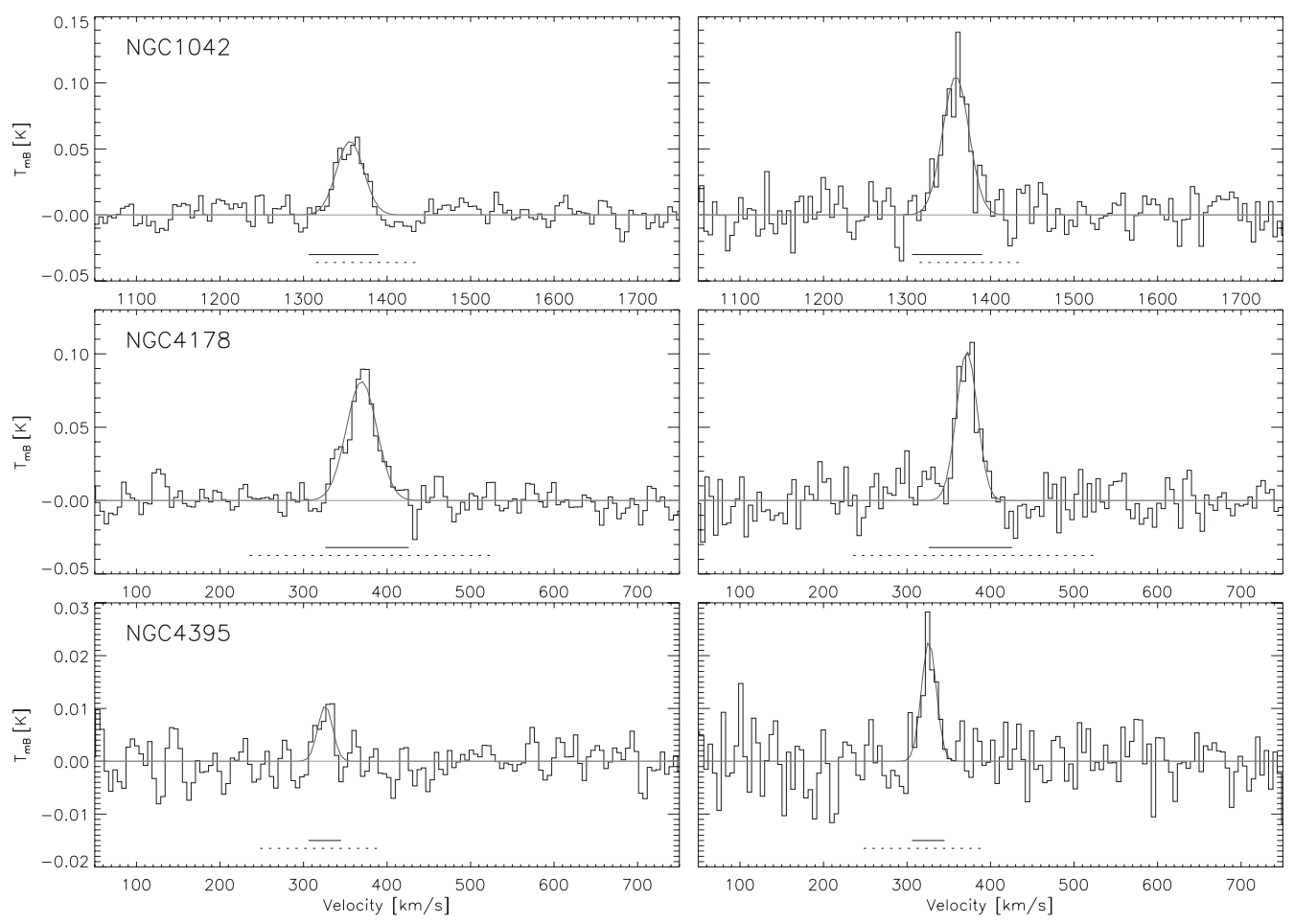

Fig. 2. ${ }^{12} \mathrm{CO}(1-0)$ (left) and ${ }^{12} \mathrm{CO}(2-1)$ (right) emission line spectra for three bulgeless spiral galaxies containing an AGN: NGC 1042 (top), NGC 4178 (middle), and NGC 4395 (bottom). The velocity resolution for all spectra is $5.2 \mathrm{~km} \mathrm{~s}^{-1}$. The horizontal lines in each panel indicate the $\mathrm{HI}$ line width at $20 \%$ level $\left(W_{20}\right.$, dashed) and the range over which the derived CO line intensities of Table 2 were integrated (solid). Also shown are Gaussian fits to the line profiles which yield an alternative estimate for the line intensities.

\subsection{CO line ratios}

A number of studies (e.g. Braine et al. 1993; Aalto et al. 1995; Papadopoulos \& Seaquist 1998) have investigated the $r_{21} \equiv$ $I_{21} / I_{10}$ ratio in galaxies, with the aim to extract information about the optical depth and excitation state of the gas. All these studies find that in the vast majority of galaxies, the value of $r_{21}$ is unity, with a relatively small scatter.

For example, Braine et al. (1993) find an average value of $r_{21}=0.89 \pm 0.06$ for a sample of 81 nearby spiral galaxies. Similarly, Aalto et al. (1995) find a mean value of $r_{21}=0.93$ in a sample of 32 starburst galaxies. Taken at face value, these results suggest that most of the molecular gas in the central regions of galaxies is optically thick and thermally excited.

However, because line intensities measured on the main beam temperature scale $T_{\mathrm{mb}}$ are equivalent to a surface brightness, and the two lines are observed with different telescope beams, interpreting the value of $r_{21}$ is difficult if the source size is unknown (Papadopoulos \& Seaquist 1998).

In Fig. 3, we compare the line intensities of the ${ }^{12} \mathrm{CO}(1-0)$ and ${ }^{12} \mathrm{CO}(2-1)$ lines in the target AGN to those in a comparison sample of morphologically similar, but quiescent, late-type spirals at similar distances and with moderate inclinations. This comparison sample was observed with the same telescope, and analyzed in the same way as the data presented here (Böker et al. 2003).

As can be seen in the right panel, the value of $r_{21}$ appears unusually high in two of the AGN (NGC 1042 and NGC 4395), while it is rather typical in NGC 4178. In this context, it is important to note that both NGC 1042 and NGC 4395 are oriented nearly face on (as is the entire comparison sample), while NGC 4178 is an edge-on spiral (see Fig. 1). Therefore, it is possible (and in fact rather likely) that the line-of-sight towards the nucleus of NGC 4178 contains gas located in its outer disk, and that our measurements for this galaxy are not really representative of the nuclear gas properties.

In order to quantify the significance level of the elevated $r_{21}$ values in the two AGN, we performed a two-Sample t-Test. This test is designed to check whether the null hypothesis (i.e. that the filled squares in Fig. 3 are randomly drawn from the same population as the open squares) can be rejected with confidence. The mean $r_{21}$ in NGC 1042 and NGC 4395 differs from the mean $r_{21}$ of the comparison sample by 4.75 standard errors, and the probability that both are part of the same parent population is 0.0001 . In other words, it can be concluded with $99.99 \%$ confidence that the two face-on AGN discussed in this paper indeed have higher $r_{21}$ values than their non-active counterparts ${ }^{1}$.

Of course, given that there are only two objects studied so far, one cannot conclude that all low-luminosity AGN observed face-on show elevated CO line ratios. Nevertheless, Fig. 3 provides tentative evidence that the $r_{21}$ value may be a good indicator for low-level nuclear activity. The reliability of $r_{21}$ in this context should be tested with similar observations of other mini-AGNs. An obvious candidate for such an observational test is NGC 3621, the only other bulge-less spiral galaxy with an AGN, which, unfortunately, cannot be observed from the northern hemisphere, but should become a prime target for ALMA.

\subsection{Effects of source size}

We now discuss some implications of the fact that we do not know a priori the spatial extent of the CO-emitting region. In principle, one can estimate the intrinsic source size using the following relation between the absolute source brightness $T_{\mathrm{s}}$ and

1 For completeness, we mention that the significance is reduced to $99 \%$ if the edge-on AGN NGC 4178 is included in the analysis. 
T. Böker et al.: Molecular gas around low-luminosity AGN in late-type spirals
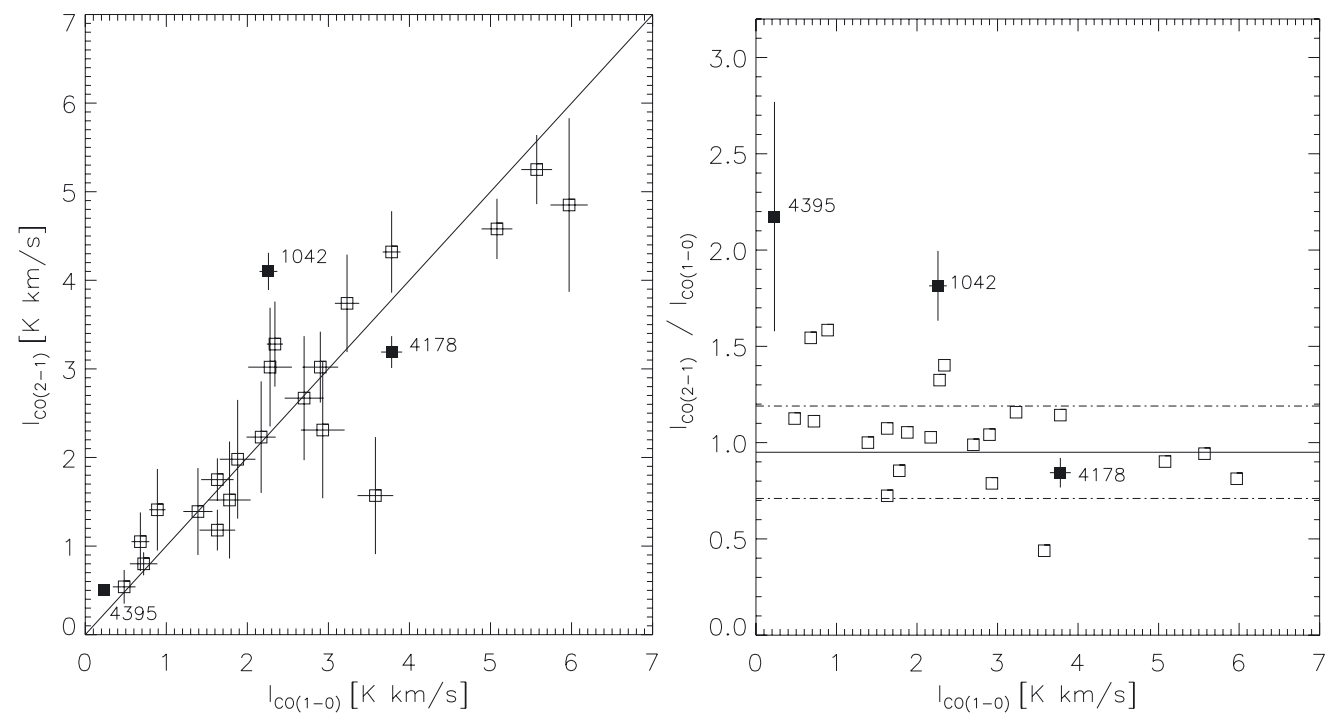

Fig. 3. Left: line intensities of ${ }^{12} \mathrm{CO}(1-0)$ and ${ }^{12} \mathrm{CO}(2-1)$ for quiescent late-type spirals (Böker et al. 2003, open squares) and those with lowluminosity AGN (filled squares, this work). Right: $I_{21} / I_{10}$ ratio vs. $I_{10}$, indicating the elevated line ratio for NGC 1042 and NGC 4395 . Here, the error bars for the comparison sample are not shown for clarity. The horizontal lines indicate the mean value of $I_{21} / I_{10}$ (solid) and the mean $\pm 1 \sigma$ (dashed) for the comparison sample.

the main beam brightness $T_{\mathrm{b}}: T_{\mathrm{s}}=T_{\mathrm{b}} \frac{\theta_{\mathrm{s}}^{2}+\theta_{\mathrm{b}}^{2}}{\theta_{\mathrm{s}}^{2}}$, where $\theta_{\mathrm{s}}$ is the source size and $\theta_{\mathrm{b}}$ the beam size.

In order to solve for the source size, we can assume for the moment that the intrinsic temperatures of the $\mathrm{CO}(1-0)$ and $\mathrm{CO}(2-1)$ line emission are the same, i.e. that $T_{\mathrm{s} 10}=T_{\mathrm{s} 21}$, which leads to:

$\theta_{\mathrm{s}}=\sqrt{\left(T_{\mathrm{b} 21} \theta_{\mathrm{b} 21}^{2}-T_{\mathrm{b} 10} \theta_{\mathrm{b} 10}^{2}\right) /\left(T_{\mathrm{b} 10}-T_{\mathrm{b} 21}\right)}$.

Assuming also that the line profiles of the ${ }^{12} \mathrm{CO}(1-0)$ and ${ }^{12} \mathrm{CO}(2-1)$ are identical, one can replace the peak temperatures with the less noisy integrated line intensities from Table 2. This results in source sizes of 16" and 12" for NGC 1042 and NGC 4395, respectively. Thus, in order to explain the observed elevated $r_{21}$ value solely by beam dilution effects, the $\mathrm{CO}$ emission cannot be more extended than this.

Unfortunately, the sensitivity of our off-nuclear spectra does not allow to place strong constraints on the actual extent of the $\mathrm{CO}$ emission, because the upper limits for the line intensities (see Table 2) are still consistent with extended emission. We therefore use the Spitzer/IRAC $8 \mu \mathrm{m}$ image of NGC 4395 (Dale et al. 2009) to check whether a compact CO source is a reasonable assumption. Because the IRAC $8 \mu \mathrm{m}$ channel is dominated by emission from large molecules, mainly Polycyclic Aromatic Hydrocarbonates, it should be a good tracer of molecular gas. Inspection of this image shows a rather compact emission region of $\approx 11^{\prime \prime}$ ( $220 \mathrm{pc}$ ) diameter surrounding the central point source. However, there is also a network of narrow and well-defined dust filaments throughout the central $1^{\prime}$ which could also contribute to the $\mathrm{CO}$ flux.

On the other hand, none of the galaxies in the comparison sample without AGN shows $r_{21}$ values as high as those observed in NGC 1042 and NGC 4395, even though they are morphologically similar and at comparable distances. Interestingly, there are four galaxies (NGC 2805, NGC 3445, NGC 3782, and NGC 5669) that fall above the $1 \sigma$ deviation. These may be candidates for follow-up searches for signs of nuclear activity, for example with deep optical spectroscopy that is able to accurately measure emission line ratios in the nucleus, similar to the case of NGC 1042 (Shields et al. 2008).

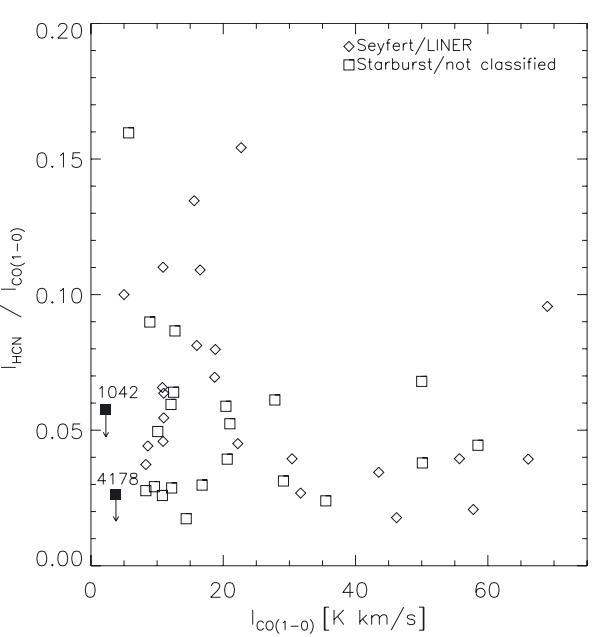

Fig. 4. Comparison of the $I_{\mathrm{HCN}} / I_{10}$ ratio in NGC 1042 and NGC 4395 to that in a sample of active (diamonds) and non-active (squares) galaxies from Gao \& Solomon (2004a).

Because there is no apparent reason why the gas distribution in NGC 4395 and NGC 1042 should be more concentrated than in the galaxies of the comparison sample, we consider it unlikely (but cannot rule out) that the elevated $r_{21}$ ratio is explained by a very compact CO source in NGC 4395 and NGC 1042. Instead, we suggest that the molecular gas in these two objects is not in thermal equilibrium, and that the $\mathrm{CO}(2-1)$ emission is excited by ionizing radiation.

This ionizing radiation can, in principle, be produced either by an AGN or by young massive stars in the NSC. However, given that most galaxies in the comparison sample also have a nuclear cluster, but do not show elevated values of $r_{21}$, it seems plausible to assume that it is indeed the AGN that is responsible for the excitation of $\mathrm{CO}(2-1)$ in these two galaxies.

\subsection{Dense molecular gas around weak AGN?}

Because the critical density for the excitation of the groundlevel of the HCN molecule is $n_{\mathrm{cr}} \approx 10^{5} \mathrm{~cm}^{-3}$, the $\operatorname{HCN}(1-0)$ 
line probes significantly higher gas densities than the ${ }^{12} \mathrm{CO}(1-0)$ line. In principle, the value of $r_{\mathrm{HCN}} \equiv I_{\mathrm{HCN}} / I_{10}$ can thus be used to estimate the average density of the molecular gas observed. This has recently been investigated in more detail by Matsushita et al. (2010) who studied the behavior of $r_{\mathrm{HCN}}$ as a function of kinetic temperature and $\mathrm{H}_{2}$ density by utilizing a Large Velocity Gradient (LVG) model. Their Fig. 5 shows that $r_{\mathrm{HCN}}$ is very sensitive to the density of the gas while being insensitive to the actual kinetic temperature. Our observed upper limits for $r_{\mathrm{HCN}}$ of 0.058 and 0.026 for NGC 1042 and NGC 4178 indicate gas densities below $1-2 \times 10^{3} \mathrm{~cm}^{-3}$.

Our derived upper limits for $r_{\mathrm{HCN}}$ in $\mathrm{NGC} 1042$ and NGC 4178 lie at the low end of the observed range of $r_{\mathrm{HCN}}$, but are consistent with both starforming galaxies and low-luminosity AGN. This is illustrated in Fig. 4 which compares the upper limits for these two galaxies to the measurements of Gao \& Solomon (2004a) who studied a sample of 53 galaxies of various types, including both active and non-active nuclei.

Taken together, this suggests that the molecular ISM in the two low-luminosity AGN studied here is similar to that in other galaxies, and certainly shows no evidence for an enhanced gas density as observed in some nearby AGN (Kohno et al. 2008; Juneau et al. 2009). Hence, we conclude that the presence of a weak AGN in NGC 1042 and NGC 4178 has no impact on the observed ISM density.

\section{Summary}

We have measured the ${ }^{12} \mathrm{CO}(1-0)$ and ${ }^{12} \mathrm{CO}(2-1)$ emission from the nuclei of three bulgeless spiral galaxies with low-luminosity AGN: NGC 1042, NGC 4178, and NGC 4395. In the case of NGC 4395, this constitutes the first reported detection of CO. The $\mathrm{CO}$ emission is rather faint, and the inferred molecular gas masses are low. This is especially true for NGC 4395 where the entire $\mathrm{H}_{2}$ mass could be contained in a single GMC.

Two of the three target galaxies (NGC 1042 and NGC 4395) show elevated values of the intensity ratio $r_{21}$. Both of these are oriented nearly face-on, and thus offer an unobstructed sight line into their nucleus. The third target (NGC 4178), in contrast, is highly inclined, and the fact that it shows a value of $r_{21}$ that is typical for other late-type spirals without an AGN may therefore be explained by its $\mathrm{CO}$ spectra being dominated by gas located in the outer disk.

We have discussed, even though we do not have a priori knowledge of the size of the CO-emitting region in our targets, that the elevated $r_{21}$ values in the two face-on AGNs are unlikely caused by beam dilution due to an extremely compact source size. This is based on estimates of the required source size that could explain the observed line ratios and comparison with the gas morphology deduced from Spitzer imaging. Instead, we argue that the elevated $r_{21}$ values are more likely caused by ionizing radiation, implying that even energetically weak AGN can impact the physical state of the surrounding gas.

We do not detect any tracers of dense molecular gas such as $\mathrm{HCN}$ or $\mathrm{HCO}+$, but the sensitivity of our observations allows us to establish meaningful upper limits. The derived gas density is less than $n_{\mathrm{H}_{2}} \approx 2 \times 10^{3} \mathrm{~cm}^{-3}$ which is at the low end of the range observed in nearby quiescent galaxies, and certainly lower than in more energetic AGN. This implies that using the value of $R_{\mathrm{HCN}}$ as an indicator for the presence of an (obscured) AGN is not reliable for weak AGN. More generally, the scarcity of dense gas in our target galaxies suggests that the conditions for star formation are poor in these nuclei, and may well be linked to their inefficient bulge growth.

Our observations have demonstrated that studies of the molecular gas around the faintest AGN are feasible, but at the limit of even the best current observatories. Looking ahead, however, these and similar galaxies should be interesting targets for future interferometers, and in particular the ALMA observatory, because its improved sensitivity and spatial resolution promises to reveal in detail the feedback mechanism(s) between the AGN, the nuclear star cluster, and the ISM in their vicinity.

Acknowledgements. We are grateful for a thoughtful referee report by Jeremy Lim, whose comments prompted a more thorough statistical analysis of our results, and helped to improve the overall quality of the paper. We are also grateful to S. Meidt and P. Jakobsen for help with identifying the correct statistical test to gauge the significance of our results. This work is based on observations with the Instituto de Radioastronomia Milimétrica (IRAM) $30 \mathrm{~m}$ telescope. IRAM is supported by INSU/CNRS (France), MPG (Germany, and IGN (Spain). We made use of the Nasa Extragalactic Database (NED) and of the Lyon Extragalactic Database (LEDA). U.L. acknowledges support by the research project AYA2007-67625-C02-02 from the Spanish Ministerio de Ciencia y Educación and the Junta de Andalucía (Spain) grant FQM-0108. U.L. warmly thanks IPAC (Caltech) for their hospitality, where this work was finished during a sabbatical stay.

\section{References}

Aalto, S., Booth, R. S., Black, J. H., \& Johansson, L. E. B. 1995, A\&A, 300, 369 Barth, A. J., Strigari, L. E., Bentz, M. C., Greene, J. E., \& Ho, L. C. 2009, ApJ, 690, 1031

Böker, T., Laine, S., van der Marel, R. P., et al. 2002, AJ, 123, 1389

Böker, T., Lisenfeld, U., \& Schinnerer, E. 2003, A\&A, 406, 87

Boselli, A., Casoli, F., \& Lequeux, J. 1995, A\&AS, 110, 521

Boselli, A., Lequeux, J., \& Gavazzi, G. 2002, A\&A, 384, 33

Braine, J., Combes, F., Casoli, F., et al. 1993, A\&AS, 97, 887

Caffau, E., Ludwig, H.-G., Steffen, M., et al. 2008, A\&A, 488, 1031

Dale, D. A., Cohen, S. A., Johnson, L. C., et al. 2009, ApJ, 703, 517

Ferrarese, L., \& Merritt, D. 2000, ApJ, 539, L9

Filippenko, A. V., \& Sargent, W. L. W. 1989, ApJ, 342, L11

Fisher, J. R., \& Tully, R. B. 1981, ApJS, 47, 139

Gao, Y., \& Solomon, P. M. 2004a, ApJS, 152, 63

Gao, Y., \& Solomon, P. M. 2004b, ApJ, 606, 271

Gebhardt, K., Bender, R., Bower, G., et al. 2000, ApJ, 539, L13

Gebhardt, K., Lauer, T. R., Kormendy, J., et al. 2001, AJ, 122, 2469

Graciá-Carpio, J., García-Burillo, S., Planesas, P., \& Colina, L. 2006, ApJ, 640, L135

Juneau, S., Narayanan, D. T., Moustakas, J., et al. 2009, ApJ, 707, 1217

Kenney, J. D., \& Young, J. S. 1988, ApJS, 66, 261

Kohno, K. 2005, in The Evolution of Starbursts, ed. S. Hüttmeister, E. Manthey, D. Bomans, \& K. Weis, AIP Conf. Ser., 783, 203

Kohno, K., Nakanishi, K., Tosaki, T., et al. 2008, Ap\&SS, 313, 279

Komugi, S., Sofue, Y., Kohno, K., et al. 2008, ApJS, 178, 225

Krips, M., Neri, R., García-Burillo, S., et al. 2008, ApJ, 677, 262

Matsushita, S., Kawabe, R., Kohno, K., Tosaki, T., \& Vila-Vilaró, B. 2010, PASJ, 62, 409

Meijerink, R., \& Spaans, M. 2005, A\&A, 436, 397

Meijerink, R., Spaans, M., \& Israel, F. P. 2006, ApJ, 650, L103

Meijerink, R., Spaans, M., \& Israel, F. P. 2007, A\&A, 461, 793

Nayakshin, S., Wilkinson, M. I., \& King, A. 2009, MNRAS, L285

Papadopoulos, P. P., \& Seaquist, E. R. 1998, ApJ, 492, 521

Peterson, B. M., Bentz, M. C., Desroches, L., et al. 2005, ApJ, 632, 799

Pilyugin, L. S. 2001, A\&A, 369, 594

Satyapal, S., Vega, D., Heckman, T., O'Halloran, B., \& Dudik, R. 2007, ApJ, 663, L9

Satyapal, S., Böker, T., Mcalpine, W., et al. 2009, ApJ, 704, 439

Schinnerer, E., Böker, T., Meier, D. S., \& Calzetti, D. 2008, ApJ, 684, L21

Shields, J. C., Walcher, C. J., Böker, T., et al. 2008, ApJ, 682, 104

Sternberg, A., Genzel, R., \& Tacconi, L. 1994, ApJ, 436, L131

Strong, A. W., Bloemen, J. B. G. M., Dame, T. M., et al. 1988, A\&A, 207, 1

Thim, F., Hoessel, J. G., Saha, A., et al. 2004, AJ, 127, 2322

Tully, R. B. 1988, Nearby Galaxies Catalog (CUP) 